\title{
Custos de produção de tilápias (Oreochromis niloticus) em um modelo de propriedade da região oeste do Estado do Paraná, Brasil
}

\author{
Nile tilapia (Oreochromis niloticus) production costs in a farm model of the west region \\ of the State of Paraná, Brazil
}

\author{
Rafael Luiz Barboza de Andrade ${ }^{1}$ Ricardo Luiz Wagner ${ }^{1}$ \\ Ilson Mahl ${ }^{1}$ Ricardo Silveira Martins ${ }^{2}$
}

\section{RESUMO}

O objetivo do estudo foi analisar os custos de produção da piscicultura praticada na região oeste do Paraná. Os custos são apurados mensalmente pela equipe do GEPEC/Piscicultura. Em linhas gerais, os custos referem-se à exploração comercial de uma área de $24.000 \mathrm{~m}^{2}$, em oito tanques, o que proporciona a produção de 14,4t de tilápia (Oreochromis niloticus) por ciclo de produção, com o peso unitário médio de $0,4 \mathrm{~kg}$. Para o custo total de implantação, a taxa de crescimento foi de 0,47\% am (ao mês) e para os custos de terraplanagem, $0,63 \%$ am, sendo que o último representa em torno de $70 \%$ dos investimentos iniciais. Os custos fixos apresentaram uma taxa de crescimento de 0,032\% am, o custo variável representou $70,18 \%$ do custo total de produção e uma taxa de crescimento de 0,32\% am, o que exige a necessidade de se verificar alternativas para diminuir esses custos, que são bastante sensíveis às variações nos preços das matérias-primas. Ficou evidenciada a necessidade do estabelecimento de um agente responsável pela governança da cadeia, para garantir sua sobrevivência.

Palavras-chave: piscicultura, custos de produção, economia agrícola paranaense.

\section{ABSTRACT}

The objective of this research was to analyze the production cost of fish production in the western region of Paraná, Brazil. The costs were obtained monthly by the GEPEC/ Pisciculture group. In general, the costs refer to the commercial exploration of a $24,000 m^{2}$ area, in eight tanks, which enable the production of 14.4t of Nile Tilapia (Oreochromis niloticus) per production cycle, with an average unit weight of $0.4 \mathrm{~kg}$. For the total cost of the implantation, the growth rate was of $0.47 \%$ pm (per month) and for the earthwork costs, $0.63 \%$ pm, representing around $70 \%$ of the initial investments. The fixed costs represented a growth rate of $0.032 \% \mathrm{pm}$, the variable cost represented $70.18 \%$ of the total production cost and a growth rate of $0.32 \% \mathrm{pm}$ which demands the necessity of verifying alternatives to decrease these costs, which are very sensitive to the variation of raw material prices. The necessity of the establishment of a responsible agent for the leadership of the food chain was exposed to guarantee its survival.

Key words: pisciculture, production costs, agricultural economy of Paraná.

\section{INTRODUÇÃO}

A piscicultura constitui-se em um moderno sistema de produção agropecuária. Como tal, para obter os lucros esperados, devem-se manejar métodos adequados e modernos baseados em princípios científicos, ecológicos, tecnológicos e econômicos (HEPHER \& PRUGININ, 1985). Projetos executados sem as devidas análises econômicas podem constituir-se num caminho curto para o fracasso (CASACA \& TOMAZELLI JÚNIOR, 2001), como por exemplo,

${ }^{1}$ Estudantes do Curso de Engenharia de Pesca da Universidade Estadual do Oeste do Paraná, (UNIOESTE), Campus de Toledo e membros do Grupo de Estudos do Agronegócio e Desenvolvimento Regional (GEPEC), Piscicultura.

${ }^{2}$ Professor da UNIOESTE, Campus de Toledo, Doutor, Coordenador Setorial do GEPEC, Piscicultura, CP. 520, 85900-970, Toledo, PR, E-mail: rsmartins@ unioeste.br. 
no caso do conhecimento e análise dos custos de operação dos projetos de cultivo de peixes (SAINT-PAUL, 1986).

No Paraná, segundo a Empresa de Assistência Técnica e Extensão Rural (EMATER, 2003), cerca de 22 mil produtores dedicam-se à atividade, produ-

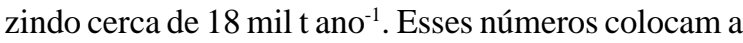
piscicultura paranaense entre os três maiores produtores nacionais, experimentado um crescimento anual médio de 37\% nas safras de 1996 a 2000 (MARTINS et al., 2001), em que foi realizada uma safra por ano.

A piscicultura é uma atividade em ascensão dentro do setor agropecuário do Estado do Paraná por constituir-se uma importante alternativa para a pequena propriedade. Porém, como ocorre com todas as novas opções de produção, sua implantação deve ser bem planejada e precedida de estudos e pesquisas que indiquem, com segurança, os melhores sistemas de produção a serem utilizados. Caso contrário, ao invés de promover o desenvolvimento regional, poderá resultar em falta de estímulo a novos investimentos e em abandono de unidades de produção. Alguns estudos têm apontado o momento crítico da piscicultura praticada na região oeste paranaense (HERMES et al., 2002; SCHREIBER et al., 2003; MARTINS et al., 2003a; 2003b; MAHL et al., 2003).

Devido à variação nos custos de implantação e produção da piscicultura, este estudo analisou os custos de produção identificando pontos de estrangulamento e possíveis soluções na busca de reduzi-los. Especificamente, trata-se de identificar a evolução dos diferentes itens da matriz de custos, bem como identificar os itens relevantes dos custos na piscicultura.

\section{METODOLOGIA}

A metodologia seguida para esta pesquisa é um estudo de caso que está amparado nos princípios da economia da produção, dos custos de produção e dos aspectos técnicos da engenharia de pesca. A teoria econômica da produção fornece os princípios básicos para análise de custos de produção.

A piscicultura e agropecuária utilizam-se de procedimentos metodológicos da administração rural para cálculo dos custos de produção. Segundo IPARDES (1992), a elaboração das planilhas de custos de produção é feita através de grandes agregados que concorrem para geração de produtos agropecuários, como: insumos, máquinas e implementos, mão-de-obra, custo financeiro, depreciação, seguro, impostos sobre a terra (ITR), imposto imobiliário, imposto sobre bens e serviços, encargos sobre salários etc.

Uma vez conhecidos e dominados os itens de custeio e as relações técnicas da produção, a presença/ausência de economias de escala pode ser um indicador importante na tomada de decisão relativa aos negócios. As economias de escala são definidas, de uma forma genérica, como sendo a relação de crescimento menos que proporcional dos custos unitários dos produtos com a quantidade produzida. O prolongamento das séries conduz a uma diminuição relativa da parte dos custos fixos dentro do custo total, proporcionando uma redução do custo unitário dos produtos fabricados (ULHARUZO, 1993).

Custos da piscicultura

O Grupo de Pesquisa em Agronegócio e Desenvolvimento Regional - (GEPEC) visa apresentar informações, análises técnicas e artigos científicos referentes às principais variáveis e indicadores relativos ao agronegócio e desenvolvimento regional do Estado do Paraná. Atualmente, o Grupo Setorial de piscicultura, no GEPEC da UNIOESTE/Campus de Toledo, vem desenvolvendo pesquisas sobre a economia da piscicultura regional através de planilhas de custo da atividade, com consultas permanentes a outros profissionais ligados à atividade, no IAP (Instituto Ambiental do Paraná), EMATER (Empresa de Assistência Técnica e Extensão Rural), e também na ATOAQUI (Associação Toledana de Aqüicultura).

Os custos são apurados nos níveis de implantação e produção, com base nos critérios técnicos da Tabela 1. Não são diferenciados entre curto e longo prazos.

Custos de implantação da piscicultura

Os custos de implantação são atualizados mensalmente pelo grupo GEPEC, referindo-se aos seguintes itens: serviços de Drenagem e Terraplanagem (trator de esteira D-50 e retro-escavadeira); materiais de construção (cano de PVC 100mm, joelho PVC $100 \mathrm{~mm}$, tijolos 6 furos, areia, pedra brita e tábuas para o monge); madeiras (viga de pinheiro - $5 \mathrm{x} 10 \mathrm{~cm}$, sarrafos - $5 \times 1 \mathrm{~cm}$ e tábuas de itauba $-2,5 \times 15 \mathrm{~cm}$ ); materiais e equipamentos (tarrafa - corrente $13 \mathrm{~mm}$, alt $2 \mathrm{~m}$, roda $113,5 \mathrm{~m}$, rede de arrasto - $12 \mathrm{~mm}$, fio $210 \mathrm{~d} / 18$, alt $4 \mathrm{~m}$, balança peixeiro e Kit para análise de água); benfeitoria (cimento, tijolos 6 furos, areia, pedra brita, madeiras vigas e sarrafos, cal hidratada e telhas francesas); mãode-obra (mestre de obra e serviços gerais); taxas e im- 
Tabela 1 - Dados técnicos para a apuração dos custos.

\begin{tabular}{|c|c|c|}
\hline & Alevinos $\mathrm{m}^{-2}$ (engorda) & 2,50 \\
\hline & Alevinos $\mathrm{m}^{-2}$ (alevinagem) & 20,00 \\
\hline \multirow{3}{*}{ Fase I (30 dias) } & Peso inicial (g) & 02,00 \\
\hline & Peso $\mathrm{p} /$ troca ração $45 \%$ para $32 \%(\mathrm{~g})$ & 30,00 \\
\hline & Conversão & 00,70 \\
\hline \multirow{3}{*}{ Fase II (60 dias) } & Peso inicial (g) & 30,00 \\
\hline & Peso $\mathrm{p} /$ troca ração $32 \%$ para $24 \%(\mathrm{~kg})$ & 00,20 \\
\hline & Conversão & 01,00 \\
\hline \multirow{19}{*}{ Fase III (90 dias) } & Peso inicial (kg) & 00,20 \\
\hline & Peso final $(\mathrm{kg})$ & 00,40 \\
\hline & Conversão & 01,40 \\
\hline & Área média do viveiro $\left(\mathrm{m}^{2}\right)$ & 3.000 \\
\hline & Numero de viveiros $\mathrm{p} /$ engorda & 7 \\
\hline & Numero de viveiros p/ produção alevino II & 1 \\
\hline & Numero total de viveiros & 8 \\
\hline & Área necessária para viveiros $\left(\mathrm{m}^{2}\right)$ & 24.000 \\
\hline & Espelho de água p/ produção de alevino II $\left(\mathrm{m}^{3}\right)$ & 2.250 \\
\hline & Espelho de água $\mathrm{p} /$ engorda $\left(\mathrm{m}^{3}\right)$ & 15.750 \\
\hline & Espelho de água total $\left(\mathrm{m}^{3}\right)$ & 18.000 \\
\hline & Produção (ciclo) & 14.400 \\
\hline & Produtividade (ha ciclo $^{-1}$ ) & 8.000 \\
\hline & Duração de 1 ciclo de produção (mês) & 6,0 \\
\hline & Números de ciclos por ano & 1,5 \\
\hline & Perdas por mortalidade $(\%)$ & 20,0 \\
\hline & Encargos sociais da mão de obra na construção (\%) & 88,8 \\
\hline & Encargos sociais da mão de obra na produção (\%) & 75,0 \\
\hline & Biometria a cada (dias): & 7,00 \\
\hline
\end{tabular}

Fonte: MARTINS et al. (2003a).

postos (licença de outorga, licença ambiental e CREA) e assistência técnica.

Custo de produção da piscicultura

Os custos de produção foram apurados com base na prática dos produtores, sendo diferenciados entre os fixos e os variáveis. Considera-se entre os Custos fixos: depreciação linear (equipamentos - viveiros e benfeitorias referentes a uma vida útil de 15 anos); remuneração do capital (equipamentos, viveiros e terra relativos ao custo de oportunidade, à base de $1 \%$ am - conforme referência para remuneração financeira de investimentos em ativos); mão-de-obra; taxas e impostos. Entre os custos variáveis estão: insumos (alevinos, ração, fertilizantes, calagem, kit para análise de água); assistência técnica e mão-de-obra temporária.

Os custos totais de produção são considerados, então, como a soma dos custos fixos e variáveis. Para efeito desta pesquisa, os valores foram atualizados para abril de 2004, pelo Índice Geral de Preços (IGP).

Os dados analisados referem-se aos custos de implantação e aos custos operacionais da atividade apurados entre agosto/2000 e abril/2004.

\section{RESULTADOS E DISCUSSÃO}

Custos de implantação

Pôde-se constatar que, após 45 meses de coletas de preços, as variações dos custos de implantação seguiram de perto o desempenho dos custos com drenagem e terraplanagem. Estes itens de custos tiveram uma participação média no período de $70,81 \%$ no custo total de implantação. Para o custo de implantação a taxa de crescimento é de $0,47 \%$ am e para os custos de terraplanagem, $0,63 \%$ am.

O menor valor para o custo de implantação registrado foi em abril de $2001(\mathrm{R} \$ 17.871,30)$ e o maior em abril de 2003 (R $\$ 23.434,89$ ), adquirindo, em abril de 2004 , um valor referente a $\mathrm{R} \$ 20.300,30$ (Figura 1). Este seria o custo do capital para iniciar no negócio, considerando-se que exista terra já disponível.

Verificou-se alta aderência destes custos ao preço do diesel e dos efeitos da elevada rentabilidade da cultura da soja, o que gera um aumento significativo nos custos em períodos de plantio.

Ciência Rural, v.35, n.1, jan-fev, 2005. 
Custos de produção

O menor custo de produção foi registrado no mês de dezembro de 2000 (Figura 1), que viabilizou o segundo maior lucro (Figura 3). O maior custo ocorreu em fevereiro de 2003 (Figura 1), que também ocasionou o mês de menor lucro da atividade (Figura 3). Ou seja, como os preços recebidos pelos produtores sofrem pequenas variações (fontes obtidas de atualizações mensais), lucro ou prejuízo é determinado pelos custos de produção. A taxa de crescimento mensal destes custos ficou em $0,24 \%$ am, impulsionada pelos itens componentes do balanceamento da ração.

Os custos fixos apresentaram pouca variação ao longo do tempo, sendo os custos de mão-deobra fixa os direcionadores desta oscilação. Estes custos caracterizam mensalmente de 25 a $30 \%$ do custo total de produção (Figura 2), gerando uma taxa de crescimento de $0,032 \%$ am, considerada baixa. Os meses de maior e menor custo foram maio de $2002(\mathrm{R} \$ 7.995,48) \mathrm{e}$ maio de 2003 ( $\mathrm{R} \$ 7.015,17)$ respectivamente.

Os custos variáveis (Figura 2) oscilaram de forma acentuada durante todo período, podendo suas maiores variações ser, na maioria das vezes, explicadas pelo aumento ou decréscimo do custo da ração utilizada. Para os demais itens dos custos variáveis, houve pouca variação. Sua análise de crescimento geométrico ficou um pouco acima do total de produção, em torno de $0,32 \%$ am, o que resulta em procurar melhores soluções para os problemas enfrentados pela atividade.
Constatou-se acentuada variação entre os componentes dos custos variáveis. Destaca-se, por exemplo, a queda do preço dos alevinos ao longo do período considerado (crescimento negativo de $0,83 \%$ am). A desistência de alguns produtores fez com que se reduzisse a demanda por alevinos, o que conduziu a um aumento da concorrência entre os fornecedores dos mesmos. Embora decrescente ao longo do tempo, os custos de aquisição de alevinos representam, em média, 9,34\% do custo total de produção.

A ração é considerada o agente direcionador do custo variável de produção, destacando-se como um importante componente dos custos operacionais, ficando em média com 52,19\% do total do custo de produção. Os valores deste item oscilam consideravelmente, acompanhando os movimentos dos produtos agrícolas, principalmente soja e milho, que são suas matérias-primas básicas. Comparativamente, os valores registrados de outubro de 2002 a julho de 2003, caracterizam em alguns meses o dobro dos custos levantados desde agosto de 2000 até setembro de 2002.

A ração utilizada nos sistemas de cultivo da região tem representado uma taxa de crescimento de $0,57 \%$ am comparado com a taxa do custo de produção de $0,24 \%$ am, o que exige a necessidade de se verificar alternativas para diminuir esses custos, que são sensíveis às variações nos preços das matérias-primas, principalmente a soja, que segue cotação internacional e também varia de acordo com alterações cambiais.

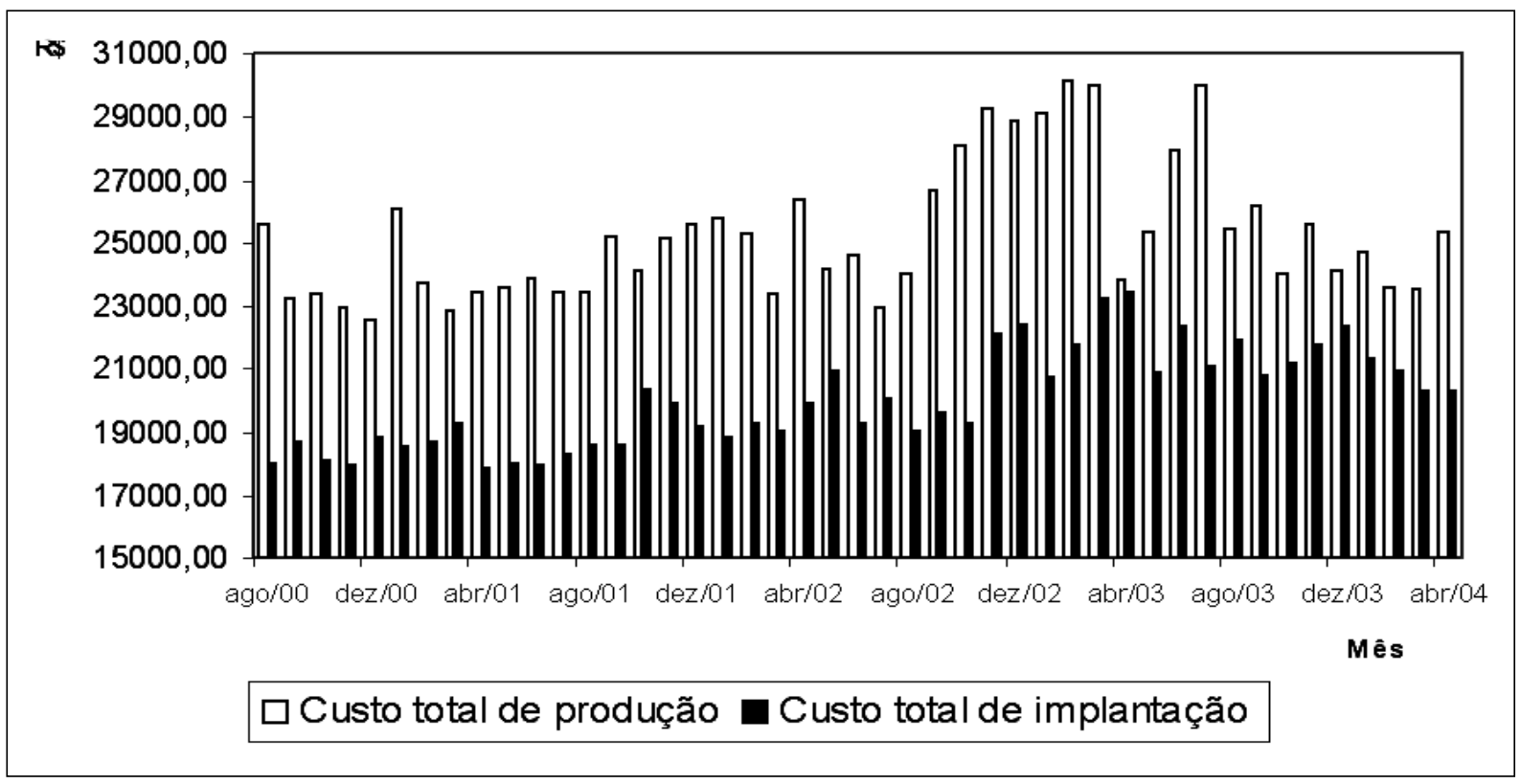

Figura 1 - Evolução dos custos totais de implantação e produção da psicultura agosto/2000 a abril/2004, a preços de abril/2004. 


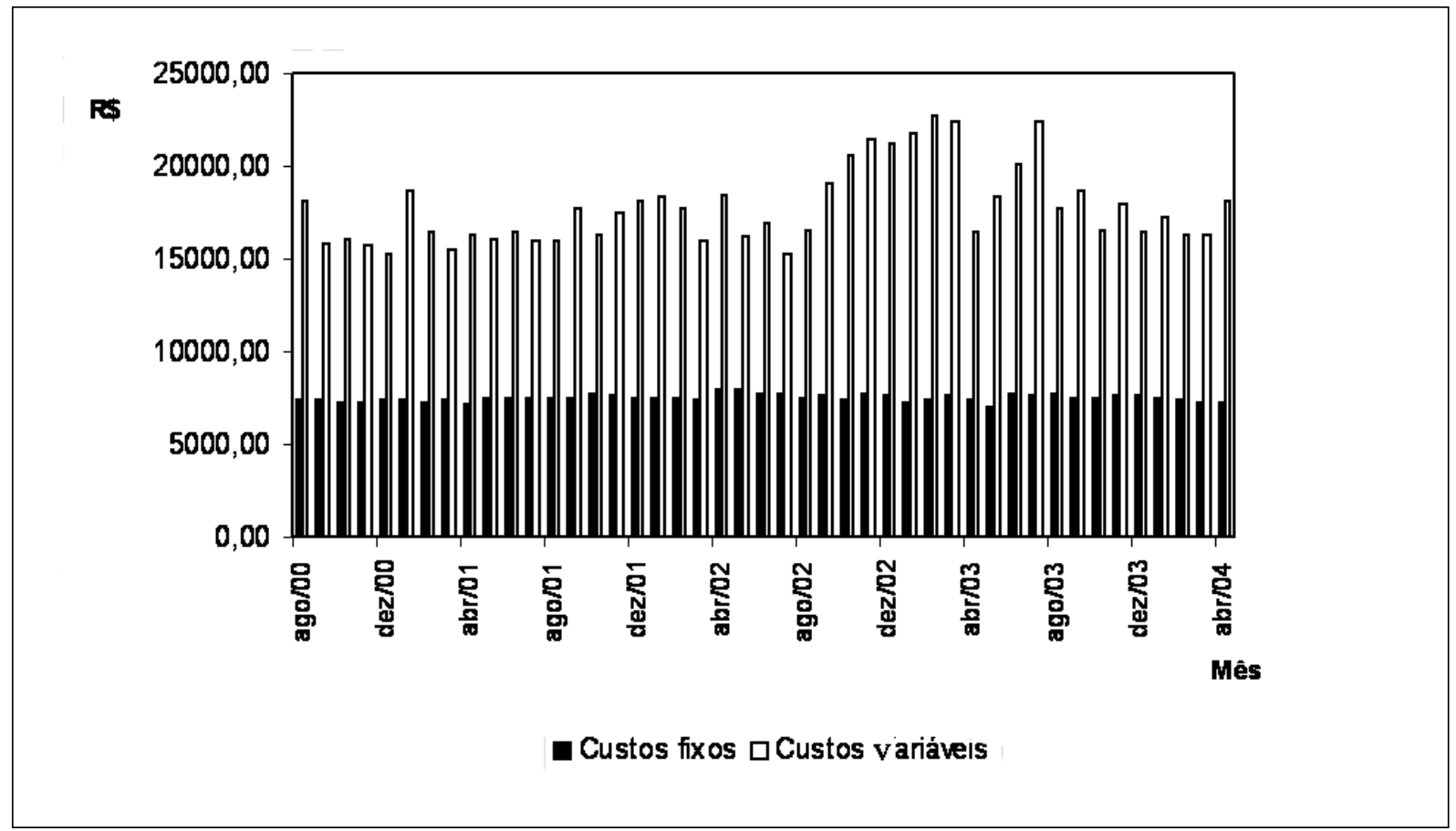

Figura 2 - Evolução dos custos fixos e variáveis da psicultura agosto/2000 a abril/2004, a preços de abril/2004

Uma forma de amenizar o problema poderia ser a formação de associações ou cooperativas que adquirissem a ração direto da fábrica em maior quantidade ou a própria associação analisar a viabilidade para estruturação de uma fábrica. Outra forma, que se pode sugerir, é fomentar a pesquisa para investigar possibilidades de se substituir certos ingredientes, preferencialmente proteicos.

F*

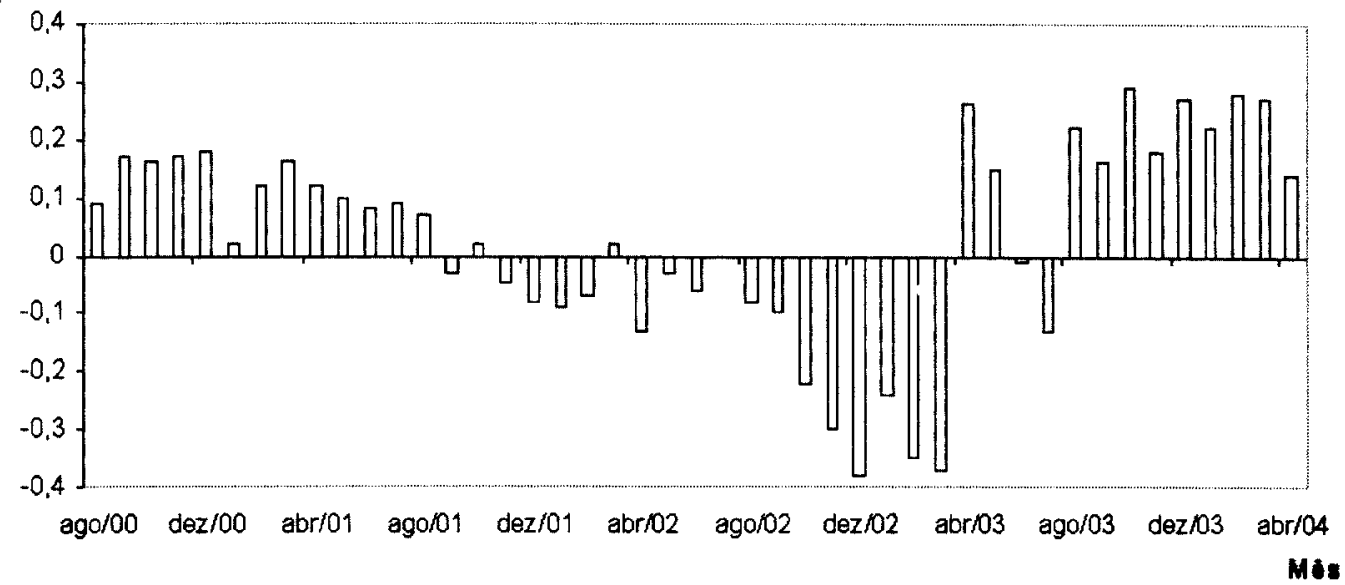

QLucratividade

Figura 3 - Evolução da lucratividade da psicultura, agosto/2000 a abril/2004, a preços de abril/2004.

Ciência Rural, v.35, n.1, jan-fev, 2005. 
Em se tratando da lucratividade da atividade, ocorreram períodos bem definidos, caracterizados por épocas de lucro e de prejuízo (Figura 3). Nos meses de agosto de 2000 a agosto de 2001, a atividade apresentou-se lucrativa, já a partir de setembro de 2001, o lucro desapareceu até março de 2003. Desde então, foi observado um lucro ascendente com posterior decréscimo, registrando, em junho e julho de 2003 novos prejuízos. O máximo e o mínimo lucro obtido foi da ordem de $\mathrm{R} \$ 0,29 \mathrm{~kg}^{-1} \mathrm{de}$ pescado em outubro de 2003 e $\mathrm{R} \$-0,38 \mathrm{~kg}^{-1}$ de pescado em dezembro de 2002.

Com base em todos os preços deflacionados, percebe-se que, nos meses de abril de 2003 a abril de 2004, o valor recebido pelo $\mathrm{kg}$ de tilápia foi o maior entre os 45 meses e, no período compreendido entre agosto de 2000 e agosto de 2002 , ocorreu acentuada queda dos preços, caindo de $\mathrm{R} \$ 1,91$ até $\mathrm{R} \$ 1,57$. Como discutido anteriormente, setembro de 2001 a março de 2003 foi um período com registro de baixo lucro ou até de prejuízo, podendo este fato ser atribuído ao baixo preço pago ao produtor.

\section{CONSIDERAÇÕES FINAIS}

Para os custos de implantação, notou-se uma certa flutuação dos preços ao longo do período. Foi identificado como principal item o serviço de máquina para implantação do viveiro, que é calculado usando o valor da hora/máquina e é dada em litros/hora (estipulado pela locadora do equipamento), com variações no preço do óleo diesel. Como direcionador dos custos variáveis, a ração se evidenciou. Sugere-se como forma de amenizar o problema, a formação de associações ou cooperativas para que adquiram ração direto da fábrica em maior quantidade, viabillizar a estruturação de uma fábrica de ração ou fomentar a pesquisa para investigar possibilidades de substituir certos ingredientes, preferencialmente protéicos.

A longevidade da atividade é dependente do desenvolvimento de esforços que visam financiar o suprimento das necessidades encontradas na implantação da piscicultura, sendo um dos principais itens o serviço de máquina para construção do viveiro. Assim, fica evidenciada a necessidade do estabelecimento de um agente responsá- vel pela governança da cadeia, para garantir sua sobrevivência.

\section{REFERÊNCIAS BIBLIOGRÁFICAS}

CASACA, J. de M.; TOMAZELLI JÚNIOR, O. Planilhas para cálculos de custo de produção de peixes. Florianópolis : Epagri, 2001. 38p. (EPAGRI. Documentos, 206). Capturado em 04 set. 2003. On line. Disponível na internet: http:// www.acaq.org.br/arquivos/docplani.pdf. EMATER. Capturado em: 03 set. 2003. On line. Disponível na internet: http:// www.emater.pr.gov.br/Piscicul/evolução.html

HEPHER, B.; PRUGININ, Y. Cultivo de peces comerciales. Mexico, DF : Limusa, 1985. 315p.

HERMES, C.A. et al. Uma análise sistêmica do agronegócio piscícola: o caso da região Oeste do estado do Paraná. Cadernos de economia, Chapecó, SC, v.6, n.11, p.99-130, 2002.

IPARDES. Paraná: desempenho macroeconômico 1992 Curitiba, 1992. 8p.

MAHL, I. et al. Análise econômica do sistema de produção de tilápias na região oeste do estado do Paraná. In: CONGRESSO BRASILEIRO DE ENGENHARIA DE PESCA, 13., 2003, Porto Seguro, BA. Anais... Porto Seguro, 2003. (CD-ROM)

MARTINS, C.V.B. et al. Avaliação da piscicultura na região oeste do estado do Paraná. Boletim do Instituto de Pesca, São Paulo, v.27, n.1, p.77-84, 2001.

MARTINS, R.S. et al. A economia da produção de tilápias em propriedades da região oeste do Estado do Paraná. In: CONGRESSO BRASILEIRO DE ECONOMIA E SOCIOLOGIA RURAL, 16., 2003, Juiz de Fora, MG. Anais... Juiz de Fora, 2003a. (CD-ROM)

MARTINS, R.S. et al. Estudo dos custos de produção e da viabilidade da produção de tilápias em propriedades da região oeste do estado do Paraná. In: ENCONTRO DE ECONOMIA PARANAENSE, 2., 2003, Maringá, PR. Anais... Maringá, 2003b. (CDROM).

SAINT-PAUL, U. Potential for aquaculture of south american fresh water fish: a review. Aquaculture, v.54, p.205-240, 1986

SCHREIBER, E. et al. Estudos para elaboração de contratos na cadeia produtiva do pescado na região oeste do Paraná. In: CONGRESSO BRASILEIRO DE ENGENHARIA DE PESCA, 13., 2003, Porto Seguro, BA. Anais... Porto Seguro, 2003. (CD-ROM).

ULHARUZO, C.G. Novos paradigmas da economia industrial e economias de escala: estudo de casos em fabricantes de autopeças do R.G.S. 1993. Monografia, Universidade Federal do Rio Grande do Sul. 